\title{
DOCUMENTOS
}




\section{ANTONIO VARGAS VEGA, INFORME RELATIVO A LAS ENSEÑANZAS UNIVERSITARIAS (1878)}

\section{PRESENTACION}

Por Gabriel Restrepo*

La Universidad Nacional de los Estados Unidos de Colombia fue originalmente concebida (1867) como "escuela de método"186. Sus fundadores buscaban enmendar los errores cometidos por los reformadores de medio siglo. Al decretar estos la libertad de enseñanza habian extendido demasiado los principios utilitaristas y se había devaluado la enseñanza oficial sin que la educación privada lograra satisfacer las exigencias de una educación racional. Dominaban el empirismo, la improvisación y la locuacidad. Allí donde la selección natural y la libre concurrencia no habían generado más que afán de saber, sin medios para realizarlo, el Estado debía proporcionar una guía, un modelo que se impusiera por su calidad en el mercado educativo.

Quienes propusieron esta reforma de estudios estaban impregnados del espíritu positivista. Estimaban 4ue el retraso del país era en buena medida imputable a la deficiente asimilación de la ciencia y de la técnica. En 1867 se hablaba de la "insipiencia en que hemos caído, precisamente cuando el resto del mundo culto ha dado pasos gigantescos en todos los ramos del saber humano"187; y en 1882 Camacho Roldán afirmaba ante un auditorio universitario: "Quedarse atrás en la carrera de las ciencias, es morir"188. Era palpable la diferencia entre la promesa acariciada por la generación de independencia y las realidades de un país devastado por la penuria y las guerras civiles. Si gracias a los esfuerzos de difusión de las ciencias realizados por Mutis a través de la Expedición Botánica podían aparecer sus discípulos Zea y Caldas con algún decoro en el escenario de las ciencias a comienzos de siglo, los progresos de éstas en el transcurso del siglo estaban fuera del alcance de las inteligencias que permanecieron en la Nueva Granada, frustradas por una organización social y educativa deficiente.

La Universidad Nacional se concibió también como factor compensatorio de integración nacional cuando la constitución federal y el caudillismo apuntaban al desarrollo de fuerzas centrífugas. Se hablaba, entonces, del "inapreciable beneficio político de entonar los ya bastantes relajados vínculos de unión nacional, mediante la hermandad de letras formadas entre jóvenes de todos los Estados"189.

En este plan los radicales pusieron demasiada confianza en el poder correctivo de la educación. La cultura en los sistemas sociales puede indicar la dirección de un cambio (como en las elaboraciones utópicas), pero su viabilidad depende de condiciones económicas y políticas que ciertamente no eran, en la década del setenta, las más adecuadas para el crecimiento del sistema educativo.

\footnotetext{
* Sociólogo, Universidad Nacional. Profesor del Departamento de Sociología de la Universidad Nacional.

186 “Qué es la Universidad Nacional", en: Anales de la Unive'rsidad Nacional, Bogotá, Tomo I, p. 6, Sept. 1868.

187 ibid., p. 4.

${ }^{188}$ Salvador Camacho Roldán, "Discurso" leído en la sesión solemne de la Universidad Nacional para la distribución de premios a los alumnos, el 10 de diciembre de 1882. En Artículos Escogidos del Doctor Salvador Camacho Roldán. Bogotá, Librería Colombiana, 1927.

189 “Qué es la Universidad Nacional”, pág. 5.

Digitalizado por RED ACADEMICA
} 
Como "Escuela de Método" o sistema metódico de las enseñanzas, la Universidad Nacional se proponía brindar el modelo de todos los escalones que componen la educación. Se incluyó una Escuela de Artes y Oficios para estimular la capacitación de los artesanos en oficios industriales que demandaban entonces la construcción de ferrocarriles y algunos proyectos industriales. La Escuela no pasaría en el período radical de los buenos propósitos, pero la idea sería retomada después de la Reforma Constitucional de 1886 con el patrocinio estatal del Instituto Salesiano.

Se con templó originalmente la necesidad de organizar por la Universidad la escuela primaria a través de un modelo de Escuela Normal, pero con el decreto del 10. de Octubre de 1870 (Ver Revista Colombiana de Educación, número 5) "quedaron deslindados los dos campos de instrucción: la primaria para las escuelas particulares i públicas; las secundarias i superior para la Universidad"190.

En su estructura la Universidad aparecía por lo tanto como un colegio mayor, y en esa forma era una prolongación de la Universidad Central, la primera universidad pública establecida en Bogotá y fundada en 1826 por Francisco de Paula Santander. Como entonces, el Colegio de San Bartolomé constituyó la base. Se añadieron las Escuelas de Jurisprudencia, de Ciencias Naturales, de Medicina y de ingeniería. A diferencia de lo que ocurrió en la Universidad Central, la Escuela de Jurisprudencia no constituía la especialidad más atractiva. La Escuela de Ciencias Naturales debía su existencia a dos fines útiles: servir de preparatorio a los estudios de Medicina y contribuir al esclarecimiento de los problemas de la agricultura. Las Escuelas de Medicina y de Ingeniería lograron afirmar estas profesiones en una dirección moderna.

En muchos estudios se ha documentado la importancia de las Escuelas profesionales $^{191}$. Poco se ha dicho, sin embargo, sobre la Escuela de Literatura y Filosofía que constituía el eje de la Universidad. De cerca de 400 estudiantes, sólo una quinta parte ascendía a los estudios superiores. Además, la Escuela de Literatura y Filosofía alimentaba la enseñanza de las ciencias jurídicas; proporcionaba instrucción básica en ciertas áreas de medicina y ciencias naturales, y daba cohesión metodológica $y$ pedagógica a la Universidad en su conjunto por el hecho de ocuparse del saber en su totalidad.

La Escuela de Literatura y Filosofía se ocupaba de los estudios generales. Preparaba a los estudiantes en los fundamentos de las artes y las ciencias, disponiéndolo racionalmente para la vida civil o para la especialización madura en una Facultad Mayor. Su plan de estudios traducía, entre nosotros, y dadas las limitaciones de nuestra angosta tradición humanista, el panorama de conocimientos que componen la formación de un individuo ilustrado: el dominio de la propia lengua; el aprendizaje del inglés, el francés y ocasionalmente el alemán, y el latín, aunque poco socorrido por los estudiantes; se impartía instrucción básica en matemáticas, geografía e historia y los principios de las ciencias naturales. A estos contenidos pro-gramáticos se añadían la gimnasia, introducida por primera vez, la música y la recreación dirigida. Se adoptaron nuevas normas de recompensas y sanciones que excluían los castigos corporales; se amplió la biblioteca y se organizó un laboratorio de física para dar consistencia a la enseñanza de las ciencias naturales.

\footnotetext{
${ }^{190}$ Anales de la Universidad Nacional. Bogotá, Tomo IV, 1870, p. 545.

${ }^{191}$ Bateman y otros, Apuntes para la historia de la ciencia en Colombia, Bogotá, Colciencias, (1975). Chaparro, Álvaro (Comp.), Ciencia y Tecnología en Colombia, Bogotá, Colcultura, 1978. 
En perspectiva, la contribución de esta Escuela al proceso educativo colombiano consistió en haber fijado las líneas directrices de los estudios secundarios. Cuando se inició, a partir de la Constitución de 1886, el paulatino deslinde entre universidad y bachillerato (propiciado por una mayor escolaridad y una diferenciación profesional), el Estado dedujo de esta experiencia la pauta de lo que prescribió como ley para los estudios secundarios tanto oficiales como privados en toda la nación. El punto más deficiente en este derrotero sería, sin embargo, la formación de profesores para la enseñanza media. La fusión de la secundaria y de la Universidad había salvado en 1867 este problema: la Escuela de Literatura contaba con la mejor inteligencia del país. Profesores de la Escuela fueron Manuel Ancízar, Miguel Antonio Caro, José María Samper, Salvador Camacho Roldán, entre otros. Sólo el establecimiento de la Escuela Normal Superior en 1935 tendió a solucionar en la enseñanza oficial este vacío.

Antonio Vargas Vega (1828-1902), el autor del documento que se publica a continuación, fue la figura directriz de este proceso. Médico y humanista, se educó como José María Samper y los miembros prominentes de la generación radical, bajo la influencia de la reforma de estudios realizada en 1842 por Mariano Ospina Rodríguez (cuyo espíritu se advierte en la vocación de la Universidad Nacional, a pesar de la distancia ideológica). Fue rector del Colegio de San Bartolomé desde 1865; Rector permanente de la Escuela de Literatura y Filosofía desde que se incorporé en 1867 a la Universidad Nacional; ejerció la Rectoría de la Universidad Nacional. Inspector del Hospital, enseñó fisiología y en esta cátedra divulgó las ideas de Claude Bernard. Ha sido reconocida su contribución al surgimiento de la medicina moderna en Colombia. Presentó proyectos de reorganización de la Universidad Nacional, sobre la Escuela de Artes y Oficios y sobre las Normales, publicados todos ellos en los Anales de la Universidad Nacional.

El Informe relativo a las enseñanzas universitarias se publicó en Bogotá en la Imprenta Galán, en 1878. Frente a la pretensión popular de recortar los estudios secundarios, el autor sostuvo la tesis de Manuel Ancízar, Rector de la Universidad, quien había afirmado con lucidez en 1870: "ninguna profesión cien tífica merece este nombre si no tiene por base completos i metódicos estudios de lo que hasta hoy se ha llamado 'humanidades' "192. El documento condensa el pensamiento y los dilemas pedagógicos que se han tratado de reseñar en estas páginas.

${ }^{192}$ Anales de la Universidad Nacional, Bogotá, Tomo IV, p. 545. 


\title{
INFORME
}

\section{PRESENTADO AL CONSEJO DE LA ESCUELA}

\author{
Por el Señor Rector de la
}

\section{ESCUELA DE LITERATURA I FILOSOFIA}

Señores Catedráticos del Consejo de Literatura i Filosofía.

Varios alumnos de esta Escuela representaron al Gran Consejo pidiendo que se les eximiera de la obligación que el nuevo Reglamento universitario les impone de ganar los cursos de Historia universal i de Retórica, Elocución i Táctica de las Asambleas, para obtener matrícula en las Escuelas superiores i optar a grado. Esta solicitud pasó al estudio del Catedrático de Contabilidad, señor Anjel María Galan, quien presentó el informe que el señor Rector de la Universidad ha sometido ahora a vuestra consideración.

El señor Galan empieza por reconocer el derecho que la Universidad tiene de modificar sus Estatutos cada vez que lo estime conveniente, i califica de laudable el propósito de obtener que las patentes universitarias sean una ejecutoria de idoneidad i no un certificado irrisorio que cubra la erudición a la violeta. Entra luego a enumerar los cursos que debe ganar un alumno para obtener el título de doctor en cualquiera de las Escuelas; i en lo tocante a los de Literatura i Filosofía, cree que el número de ellos es escesivo, i propone que los 17 que hoi se exijen para el grado superior se reduzcan a 12, o con mas propiedad a 13, pues el señor Catedrático propone que se incluya el curso de Contabilidad mercantil i oficial (que según el Reglamento vijente no es obligatorio sino para el grado de Licenciado en derecho) entre los de forzoso estudio para los alumnos que sigan las carreras de Naturalista, Médico o injeniero.

Apoya el señor Galan su proyecto de reducir no solo los cursos exijibles para el grado de doctor, sino las enseñanzas universitarias, en dos razones principales: 1.a Que exijir 17 cursos de Literatura i Filosofía para conceder la patente es rodear de tantas trabas la coronación de las mencionadas carreras, que sólo los hijos de los hombres que tengan medios i paciencia para sostenerlos diez años en la Universidad podrán conseguirla. "Es, dice, cerrar las puertas a las aspiraciones lejítimas de muchos jóvenes que quisieran volver al seno de su familia con el diploma de doctor emitido por la Universidad, i que no podrán hacerlo porque sus padres no pueden sostenerlos en la capital de la República sino por 5 o 6 años; es pues volver a los tiempos en que solo una clase de la sociedad podia aspirar a tan costosa distinción." 2.a Que es conveniente "simplificar los estudios hasta donde sea posible para poner los grados universitarios al alcance de todos o del mayor número de los alumnos que deseen obtenerlos."

Por mas estrañas que parezcan estas frases, que traen a la memoria otros tiempos i otras luchas, conviene, sinembargo, poner en relievo una vez más los inconvenientes 
prácticos de tales ideas, i fijar los principios que, en mi concepto, deben tenerse presentes en todos los asuntos relacionados con las enseñanzas universitarias.

Sea lo primero hacer notar lo que tantas veces se ha repetido en el seno de este mismo Consejo, a saber: que la colación de grados i la enseñanza universitaria son dos cosas esencialmente distintas, i que deben separarse en absoluto si se quiere que la Universidad sea realmente un centro de luces en el país. La Universidad debe, a mi modo de ver, enseñar las materias que sean de su competencia, con la solidez i estension que crea necesarias para impulsar el movimiento intelectual del país, sin averiguar silos que se dedican al estudio de un ramo de las ciencias solicitarán mas tarde el diploma de aptitud. La ciencia por la ciencia i por las ventajas que de ella reportará la Nación, debe ser su divisa; i no la ciencia encaminada a la espedición de patentes de doctor.

Si se cree que el diploma es un estímulo para el estudio i una garantía para la sociedad, debe determinarse qué materias de entre las que el Instituto enseña, son las necesarias para calificar la idoneidad de los educandos en una carrera o profesión, pero esto no implica la necesidad, ni ménos la conveniencia de suprimir de la enseñanza universitaria todos aquellos ramos del saber humano que no se hallen comprendidos en la lista que se fije.

Los hombres de la jeneracion actual hemos heredado las tradiciones universitarias de la época de la Colonia i las de la República en sus primeros años de existencia. Entónces se exijian títulos académicos para desempeñar ciertos empleos i para ejercer las profesiones de médico, abogado o sacerdote, únicas que se conocian en el país, i era lójico que la enseñanza universitaria no tuviera otro objetivo que formar doctores en aquellas privilejiadas carreras. Vino de aquí la necesidad de fijar el tiempo que debia consagrarse al estudio de cada materia, la de reducir este tiempo al mínimun posible, i la de abatir el nivel de la instruccion al mínimun reglamentario.

Ya se comprende que en la situacion presente del pais, cuando los grados académicos no son necesarios para el ejercicio de ninguna industria, ni habilitan para empleos públicos, í pueden ser espedidos por cualquier individuo o Corporacion, la Universidad obraria abiertamente contra el espíritu que presidió a su fundación si redujera sus enseñanzas a las estrictamente indispensables para el ejercicio de las cuatro profesiones que he mencionado. Los errores de esta medida serian aún mas graves i patentes si, como lo pretende el señor Galan, se cercenaran las enseñanzas hasta el límite indispensable "para poner los grados universitarios al alcance de todos o del mayor número de los alumnos que deseen obtenerlos."

¿Valdria la pena de que la Nacion entera continuara gravando su escaso Tesoro con los únicos fines de formar mediocres doctores, i de sostener un Instituto que estaria apénas al nivel de cualquier Colejio de Estado? Las patentes que, a costa de injentes sacrificios de la Nacion, espidiera la Universidad ¿serian al ménos una garantía de idoneidad que el pais entero podria aceptar con entera confianza?

A mi modo de ver, una de las causas que mas han contribuido a que no se hayan fijado aún las ideas respecto al número i la calidad de las enseñanzas universitarias, es que se han confundido en un mismo plan la enseñanza secundaria i la profesional. 
En todos los paises cultos la enseñanza profesional se rije por reglamentos especiales i forma un ramo aparte que tiene sus peculiares exijencias. Los Gobiernos protejen i sostienen a veces institutos profesionales de medicina, artes, agricultura, minería, metalurjia, injeniería civil $\boldsymbol{\&}^{\mathbf{a}} \boldsymbol{\&}^{\mathbf{a}}$, donde se enseñan los ramos de inmediata aplicacion a esta clase de conocimientos; i exijen para la matriculacion en tales escuelas pruebas de aptitud que consisten, o en el exámen del bachillerato, o en el de un conjunto de materias bien definidas i cuya estension i comprension están especificadas en los respectivos programas. El alumno tiene que contestar a tal o cual programa, o a tales o cuales proposiciones del programa jeneral de una ciencia dada, segun que solicite matrícula en una u otra de las facultades superiores.

En los Institutos de esta clase lo único que se averigua es si el alumno tiene el desarrollo mental suficiente o posee los conocimientos indispensables para comprender las materias que va a cursar, sin preocuparse de que haya aprendido estas materias en uno o en tres años o en un orden determinado de antemano.

Con este sistema, que solo exíje completa idoneidad en los estudios prévios, la instruccion superior puede reglamentarse con entera independencia de la secundaria, i se está en capacidad de darle a éste el desarrollo necesario para satisfacer las necesidades del pais o las de los padres de familia, sin sujecion a las estrechas exijencias de determinada industria o profesion.

La estraordinaria estension que han alcanzado las ciencias en este siglo, unida a la gran multiplicidad de las industrias, ha cambiado completamente la faz de la enseñanza pública. La centralizacion en las Universidades de todos los ramos de los conocimientos humanos es ya obra imposible, i para dar cabida a las variadas exijencías de la instruccion profesional e industrial ha sido indispensable crear Institutos especiales para cada ciencia o ramo nuevo de industria.

La tendencia jeneral de la época es, en suma, a especializar los estudios: de aquí la fundacion de escuelas de aplicacion al comercio, a la minería, a las ártes industriales, a la pintura, a la escultura, a la zootecnia, a los caminos, puentes i calzadas, i a otros ramos que entraban en el cuadro de las antiguas Universidades europeas. El programa de éstas ha quedado reducido a la enseñanza de la Medicina, del Derecho i de algunos ramos de las ciencias naturales, tales como la Física i la Química. A esta lista hai que agregar la Teolojía, que forma parte del plan de estudios de ciertas Universidades inglesas.

En las escuelas especiales es donde únicamente se dictan, con todos los desarrollos que la materia requiere, los ramos de enseñanza secundaria inmediatamente aplicables a la respectiva profesion o industria. Citaré algunos ejemplos. En las Escuelas de comercio se enseña de un modo especial, i con todos sus detalles, no solo las operaciones peculiares de los Bancos, Casas de comercio \&, ${ }^{a}$ sino los procederes de la Contabilidad mercantil $i$ todo lo relativo a las pesas, medidas i monedas que se usan en las principales naciones del mundo. Este aprendizaje es obra de un año por lo ménos de estudio i de práctica, i no puede, por consiguiente, comprenderse en el curso escolar de Aritmética que se dieta en establecimientos de instruccion secundaria por el estilo de la Escuela de Literatura i Filosofía de nuestra Universidad.

En las mismas Escuelas el curso de Jeografía comercial comprende un cúmulo de nociones detalladas i completas sobre las vías de comunicacion, las líneas telegráficas i de vapores, los productos industriales i agrícolas, el movimiento de los principales 
mercados, el monto de las esportaciones e importaciones, i demas puntos relacionados con el comercio de los diferentes países del mundo, nociones todas que seria imposible inculcar a los jóvenes en los diez meses que consagran al estudio de la Jeografía universal en la Escuela de Literatura i Filosofía.

El estudio de la Jeografía física, o sea de la Física del globo, ha adquirido en esta época una importancia de primer órden, puesto que su objeto es el conocimiento de la tierra en relacion con los hombres, los animales i las plantas que la pueblan; i apénas bastarian tres o cuatro años de enseñanza para hacerlo completo, i eso en el supuesto de que los educandos poseyeran las nociones prévias que la materia requiere.

En iguales consideraciones podria entrar acerca del aprendizaje del Dibujo, de la Jeometría, de la Física i aun de la Aritmética tal como debe hacerse en las Escuelas de Artes i Oficios i en la Injeniería, pero me limito a los ejemplos anteriores para llamar la atencion del Consejo a estos dos hechos: 1.o Que los Institutos especiales o de aplicacion son los encargados directamente de darle a la instruccion secundaria o preparatoria una comprension determinada i práctica; i 2.o Que la enseñanza secundaria que no tiene en mira la aplicacion inmediata a un ramo determinado de industria o a una profesion, debe ser esencialmente jeneral, o mejor dicho, debe comprender lo que cada ciencia tiene de fundamental o de universal aplicacion.

Con sobra de razon los lejisladores de nuestro pais dispusieron que las Escuelas de Artes i Oficios i la de Injenieríá civil i militar se organizaran aparte de la Universidad. De este modo se ha simplificado el programa de enseñanzas de la Escuela secundaria de Literatura i Filosofía i puede darse ya mayor cohesion a los estudios i un desarrollo mas completo a la enseñanza jeneral que en ella se dicta. Aquellas escuelas especiales han quedado por el mismo hecho en capacidad de organizar debidamente las enseñanzas preparatorias que necesitan sus educandos, sin sujetarse a los programas necesariamente incompletos de la Escuela de Literatura.

Comprendida de este modo la enseñanza secundaria, queda por investigar qué ramos debe comprender i cuál será la estension que habrá de darse al estudio de cada uno.

III

En mi opinion una buena enseñanza secundaria debe comprender aquellos ramos de la Literatura i de las ciencias que sin tener aplicación directa a una carrera determinada, sirvan de preparacion útil para todas, al mismo tiempo que de jimnástica para desarrollar el espíritu de la juventud i levantar el nivel jeneral de las intelijencias en la nacion.

Debe tenerse presente que la mayoría de los padres de familia que envian sus hijos a la Universidad solo pretende proporcionarles a éstos aquella instruccion jeneral i variada que debe tener todo hombre que aspira a ocupar un lugar digno en la sociedad. En efecto, de 400 jóvenes que concurren anualmente a las clases de Literatura i Filosofía de la Universidad, puede afirmarse que solo una $5^{\mathrm{a}}$ parte pretende sériamente coronar una de las cuatro carreras profesionales que les brinda el Instituto: el resto busca en otras ocupaciones los medios de subsistencia que en vano solicitaria del ejercicio de aquellas profesiones.

Si el fin que debe proponerse la Universidad es completar la obra de la Escuela primaria i formar, mediante una enseñanza mas completa que la que aquella da, 
jeneraciones de ciudadanos instruidos i capaces; silo que se trata es de emancipar las almas de la ignorancia i del error i fundar sobre bases sólidas la República, es indispensable que en vez de cercenar i de abatir los estudios secundarios, les demos el desenvolvimiento que cumple a los altos propósitos del Instituto.

No olvidemos que en las Repúblicas, todos los hombres están llamados a desempeñar los empleos públicos i a tomar parte mas o ménos activa en el Gobierno de la comunidad; i en ellas es por lo mismo mas imperiosa la necesidad de difundir una sólida instruccion en .todas las clases sociales, i principalmente en las que habrán de encargarse algun dia del apostolado de las ideas i de la direccion de las masas ignorantes.

En este sentido, el programa de la enseñanza universitaria, léjos de pecar por esceso, es a mi ver, en estremo diminuto, i acaso podria calificarse de vergonzante.

La enseñanza secundaria en un Jimnasio oficial de Berlin comprende las siguientes materias: Relijion, Hebreo, Griego, Latin, Frances, Aleman, Inglés, Historia patria i universal, Matemáticas (Aritmética superior, Aljebra, Jeometría), Jeografía, Física, Filosofía, Dibujo i canto. El aprendizaje de estos ramos se hace en órden ascendente, en las seis clases en que está dividida la enseñanza de cada materia (Rendu-'L'education populaire dans l'Alemagne du Nord" páiína 387).

En Francia la enseñanza secundaria de los liceos oficiales comprende los siguientes ramos: Frances, Lenguas vivas estranjeras (Inglés, Aleman, Italiano, Español o Portugues, segun la situacion jeográfica del respectivo departamento escolar), Historia Patria, Jeografía, Matemáticas, Historia natural, Hijiene, Física, Química, Contabilidad, Elocucion i Retórica. Lejislacion usual, Mecánica, Cosmografía, Moral, Economía rural, industrial i comercial, Dibujo, Jímnástica i Canto. Estos estudios se hacen en cuatro años escolares, ademas del año preparatorio; "i en cada año se da, o una parte de cada materia, o se enseñan éstas en serie ascencional i no en toda su estension." (Programes officiels de 1' enseignement secondaire spécial." Edicion oficial de 1868, pájina 2." i siguientes).

En los Institutos de enseñanza secundaria de Escocia, que es talvez la seccion del Reino Unido donde están mejor reglamentados los estudios, el currículum de éstos se divide en dos grupos segun que se aspire al bachillerato en artes o en ciencias. El primero comprende el Latin í el Griego, la Lójica, la Filosofía, la Literatura inglesa, las Matemáticas, la Física i la Astronomía en la estension fijada por los programas. El 2.0 grupo comprende las ciencias puras, o sea: las matemáticas (la Jeometría, la Trigonometría elemental i las Secciones cónicas, el Aljebra \&. ${ }^{a}$ la Física, la Química, la Jeolojía, la Botánica i la Fisiolojía animal; i las ciencias aplicadas, o sea: ya las Ciencias naturales (Botánica, Jeolojía i Zoolojía); ya las ciencias esperimentales (Química i Física) - las ciencias mecánicas (Mecánica e Injeniería civil); de acuerdo con los programas especiales para este grado, i a eleccion del postulante, eleccion que depende de los estudios profesionales a que quiera consagrarse. (Demogeot et Montucci "De 1' enseignement superieure en Angleterre et en Escosse," 1870_pájinas 470 i siguientes).

Podriamos multiplicar las citas; pero bastan las anteriores para hacer resaltar dos hechos: 1.o la importancia que se da en los países cultos a la enseñanza secundaria, i 2.0 el cuidado que se pone en la subdivision o gradacion de los cursos correspondientes a una misma materia. 
Si la pobreza del país i la índole de nuestra sociedad no permite que se dé, por ahora, a la educacion secundaria la estension que ha alcanzado en aquellos paises, i si tampoco podemos fundar escuelas de aplicacion bien dotadas í servidas para formar agricultores, comerciantes, mineros, mecánicos, pintores de primera clase $\& .^{a} \&{ }^{a}$ juzgo que debemos conservar por lo ménos el mui modesto programa de nuestra Escuela de Literatura i Filosofía, con las modificaciones que adelante indicaré.

\section{IV}

Los defectos de ese programa no consisten en la eleccion de las materias que deben constituir el conjunto de la enseñanza, sino en el corto tiempo que se destina al estudio completo de casi todos los ramos que comprende, i en la falta de gradacion de estos estudios.

Exijimos que los alumnos aprendan en nueve meses una ciencia o un arte en todos sus detalles; i como en efecto algunos jóvenes logran en tan corto tiempo, i aun en ménos, encomendar a la memoria i esplicar medianamente el contenido de un texto, nos declaramos satisfechos, i no solamente certificamos i recomendamos la perfecta competencia o idoneidad de tales educandos, sino que hacemos coro con los que proclaman que la division de ciertas materias en dos o mas cursos es cosa supérflua, i que cualquier ramo de las ciencias puede aprenderse con suficiente perfeccion en un año escolar.

Hai sin duda jóvenes que con solo los nueve meses de estudio pueden contestar satisfactoriamente a cualquier pregunta relativa al texto estenso de Física de Ganot, al de Gramática de Bello o al de Aritmética de Bourdon; i aún podria citar con sus nombres propios a varios jóvenes que han ganado en un año todos los cursos de Jurisprudencia; pero ¿puede razonablemente esperarse que de aquella indijesta aglomeración quede algo de provecho en la mente? En mi concepto, éste ha sido uno de los más graves errores de la enseñanza universitaria. En efecto, hemos adoptado estensos i mui minuciosos programas de cursos, en los cuales parece que nos hubiéramos preocupado mas del brillo que de la solidez; en muchas clases hemos calcado las lecciones sobre los textos mas nuevos i detallados, i obligamos a los discípulos a que los aprendan en el corto espacio del año escolar; i acaso nos hemos fijado demasiado en el lucimiento de los exámenes i en satisfacer al público i a los padres de familia, i hemos descuidado lo sustancial i positivo de la enseñanza.

De todo esto ha resultado un hecho que todos palpamos, a saber: que de ciertas clases universitarias, rejentadas por distinguidos profesores, no han salido hasta hoi sino mui raros alumnos realmente aprovechados; los demas han olvidado con la misma facilidad con que lo aprendieron, lo que el texto i el profesor hicieron espejear a su vista durante el corto tiempo de los estudios.

Los señores Catedráticos de los cursos superiores de la Escuela de Literatura i Filosofía i los de las Escuelas de Medicina e Injeniería han podido apreciar bien la escasa dósis de conocimientos que de las clases inferiores llevan los alumnos, aun los condecorados i sobresalientes; i los mismos señores profesores de estas clases tienen a menudo que lamentar la obligacion en que están de seguir el programa detallado de una materia en el corto espacio de un año escolar. 
Esforcémonos en hacer calar en los espíritus la idea de que la única enseñanza provechosa es la que se hace lenta i metódicamente, porque es la única que consulta la naturaleza humana. El espíritu del hombre es como su organismo físico, que no puede asimilarse ninguna sustancia que no haya sido ántes elaborada con cuidado i sometida a una lenta i laboriosa dijestion. Las opilaciones intelectuales no conducen a la ciencia, ni las opilaciones físicas al vigor i la salud.

Los miembros antiguos de este Consejo hemos venido luchando hace mucho tiempo contra la festinacion casi vertijinosa de los estudios secundarios de la Universidad, porque sabemos por esperiencia que a ella i no a las aptitudes de los alumnos se debe lo mediocre de los frutos cosechados por el Instituto.

Para patentizar lo dicho, entraré en algunos pormenores. La enseñanza entera de la Física se hace en la Escuela de Literatura en un año, i el programa comprende, naturalmente, no solo las nociones meramente elementales que son del resorte de las escuelas primarias de segundo i tercer grado, que han debido precederle, sino tambien la demostracion de las leyes jenerales de los fenómenos i las aplicaciones particulares de estas leyes. La enseñanza secundaria tiene que elevarse hasta allá, so pena de dejar al alumno en incapacidad de aprovechar en los estudios superiores.

En los Institutos de enseñanza secundaria en Francia, el estudio de este ramo se divide así: "Primer año. Nociones jenerales sobre las propiedades mas importantes de la materia (la pesantez, la densidad, la presion, la compresibilidad i la elasticidad) tomando como punto de partida fenómenos conocidos de los alumnos. Partiendo de estos datos, el profesor esplica la fusion, la ebullicion, la conductibilidad; demuestra esperimentalmente el principio de Arquimédes; esplica el uso del barómetro, del termómetro i de la máquina neumática, i termina por los fenómenos elementales de la electricidad. Las demostraciones no deben deducirse de las propiedades, consideradas de una manera abstracta, sino de los problemas presentados a Arquimédes por el rei Heron, a Galileo por los fontaneros de Florencia $\& .^{a}$

Segundo año. Propiedades jenerales de los cuerpos: líquidos, calor, electricidad. Enseñanza sencilla i puramente esperimental, sin fórmulas aljebráicas.

Tercer año. Calor, acústica, luz; repaso de electricidad dinámica. Enseñanza segun el plan de los años anteriores.

Cuarto año. Revision i complemento de los estudios anteriores. Demostracion de algunas teorías, como la del calor, de la luz, de los instrumentos de óptica i de acústica. Si es posible se hacen aplicaciones a las industrias nacionales, tomando ejemplos determinados i sometiendo a cálculos numéricos los elementos de una operacion industrial." ("Programmes officiels," pájina 3 i siguientes).

La Historia patria, la universal, la Filosofía, no comprenden en nuestra escuela sino un solo curso, i es sabido que a ménos de abatir estas enseñanzas al nivel de las que se dictan en las escuelas primarias, poco o ningun fruto sacarán de ellas los alumnos, si el programa tiene la pretension de abarcar toda la ciencia.

En los grandes centros de educacion en Europa se considera la Historia universal como un ramo de instruccion superior que no está al alcance de los jóvenes que entran a los establecimientos secundarios de educacion; i en éstos solo se enseñan las nociones indispensables para comprender la Historia patria. 
La enseñanza de este último ramo, así como del de la Filosofía se distribuye en los años o cursos que comprende el curriculum de estudios del Colejio.

Tanto en Inglaterra como en Alemania i los Estados Unidos del Norte, la enseñanza de la Filosofía en los establecimientos bien reputados, comprende no solo la contemplacion i el estudio del hombre interior sino el conocimiento del ser humano en relacion con los ajentes que lo rodean i con los demas seres que pueblan la tierra, i la historia o filiacion de sus ideas desde los tiempos prehistóricos, hasta la época actual. La Antropolojía se considera hoi como única base sólida de la Filosofía, 1 el preliminar indispensable de todo estudio relativo a esta ciencia.

Idénticas observaciones podria hacer respecto del aprendizaje de la Aritmética, de la Jeografía i de los Idiomas estranjeros; pero sé bien que mi insistencia seria superflua, puesto que hablo al Consejo que ha solicitado repetidas veces la division de estos cursos i de los programas respectivos.

En conclusion: el problema de la fijacion de los cursos de la Escuela de Literatura i Filosofía no puede resolverse, en tanto que no se haya decidido cuáles deben ser el objeto i las tendencias de los estudios de la Escuela. Si lo que con ella se propone es preparar a los alumnos para grados superiores que sean accesibles a todos o al mayor número de los que lo solicitan, i se entiende por esto que la instruccion debe comprender únicamente las materias directa o inmediatamente aplicables a la Medicina, la Injeniería, las Ciencias naturales i la Jurisprudencia, es evidente que deben reducirse los cursos no solo al número que solicita la Comision, sino a uno mucho menor; y que, ademas, conviene simplificar los programas para que la enseñanza no pase de los elementos mas sencillos e indispensables.

De este modo se acortará el tiempo de los estudios, y los cursantes obtendrán la ventaja de aprender con alguna perfeccion la escasa dósis de ciencia que les imparta la Universidad.

$\mathrm{Si}$, por el contrario, el objeto de la enseñanza en la Escuela de Literatura i Filosofía es formar caractéres i difundir en el pais los conocimientos de mas universal 1 útil aplicacion a la obra del progreso; si no se trata de formar turbas de charlatanes, o segun la espresion del señor Galan, de eruditos a la violeta, es indispensable que le demos a la enseñanza secundaria un lugar, sino tan ámplio como el que tiene en todos los paises cultos, por lo ménos el que el atraso de nuestra sociedad reclama, i el que nuestros módicos recursos autoriza.

Consideradas las cosas desde el segunto punto de vista, juzgo que no habria exajeracion en dividir las enseñanzas de Historia patria, de Historia universal, de Frances e Inglés inferiores, de Aritmética, de Física i de Jeografía en dos cursos por lo ménos, como se ha hecho ya cón la clase de Castellano, ni en establecer para las enseñanzas del Inglés i del Frances un curso superior práctico, o sea de ejercicios i de composicion como el que se estableció para el inglés en los principios de la Universidad. Es bien entendido que no pretendo con esto abogar en favor del sistema vijente de dividir un texto en dos mitades i de hacer que la clase inferior estudie la primera mitad i la superior la segunda. En una enseñanza progresiva o, mejor dicho, que procede de lo simple a lo complicado, los alumnos deben abarcar desde el principio el conjunto entero de una ciencia o arte en lo que tiene de mas jeneral i comprensible; i los ulteriores desarrollos deben ser graduados, de manera que las cuestiones abstractas 1 los problemas compli 
cados vengan despues de las nociones prácticas mas elementales que sirven de base al arte o a la ciencia que se estudia. Salta a la vista lo absurdo del procedimiento de un Institutor que pretendiera hacer un curso gradual de lengua castellana para niños, i con este fin dividiera el texto estenso del señor Bello en tres o cuatro partes, e hiciera que los niños estudiaran sucesivamente $\mathrm{i}$ en un tiempo determinado cada una de estas partes. ¿Podria llamarse gradual í metódica a esta enseñanza?

\section{V}

La institucion del Doctorado es en nuestra Universidad, 1 ha sido siempre en todos los institutos de esta clase, la causa principal de la instabilidad de las leyes i los decretos que reglamentan los estudios. Por una parte las reclamaciones de algunos superiores i catedráticos que desean conservar a todo trance la popularidad del establecimiento aun a costa de la solidez de la instruccion, i por otra parte las exijencias de muchos padres de familia que aspiran a que se acorte el término de los estudios a fin de que sus hijos "vuelvan pronto al seno de sus familias con el diploma de Doctor emitido por la Universidad," producen en el réjimen de los estudios una serie de acciones i reacciones que perjudican a los jóvenes i refluyen contra el buen nombre del instituto.

Fácilmente se concibe que en los países donde la espedicion de títulos es un monopolio atribuido a determinados establecimientos oficiales, i donde estos títulos se exijen para el ejercicio de ciertas profesiones, los dueños de tal monopolio busquen los medios de acortar los estudios i de facilitar la adquisicion de la indispensable patente; pero entre nosotros las circunstancias son totalmente diversas. Aquí pueden espedir, i espiden diariamente, títulos de Doctor no solo los colejios públicos i privados sino (como se han visto algunos ejemplos) reuniones de amigos que, en són de Junta de examinadores, convocan los postulantes: cualquiera ciudadano, haya o no haya estudiado, puede ejercer la profesion que sea mas de su agrado, i apellidarse Doctor, i fijar carteles anunciando prodijios sin que nadie le pregunte dónde ni cómo ha adquirido aquellas tan ponderadas aptitudes. Siendo esto así, no se alcanza a comprender la razon que pudiera tener la Universidad nacional para rebajar sus enseñanzas, festinar sus.estudios i limitar la instruccion que da, a nociones superficiales i deficientes, tan solo para investir pronto a sus e.ducandos con un título que no necesitan i que pueden, ademas, darse ellos mismos u obtener sin esfuerzo alguno de amigos complacientes. Ménos se alcanza a comprender cómo, en un país así constituido, pueda considerarse como indicio de la aspiracion a renovar los tiempos "en que solo una clase de la sociedad podía pretender a tan costosa distinción", el hecho de exijir ciertas pruebas de idoneidad para estender patente en nombre de la República.

Los alumnos de la Universidad pueden, si tal es su deseo, estudiar la mitad o la décima parte no mas de los cursos escolares, u optar al título de Licenciado para apellidarse luego doctores, i doctores de la Universidad. ¿Qué lei nacional o de los Estados, o qué reglamento universitario les impide hacerlo?

Hai, ademas, otro hecho hácia el cual llamo especialmente la atencion de los señores Catedráticos, i es el siguiente: todo individuo, venga de donde viniere, haya o no estudiado, puede seguir los cursos de la Universidad i asistir a las clases que quiera, con solo pedir de palabra o por escrito una boleta de permiso al Rector de la Escuela. La única condicion que se le exije es que respete el órden de la Escuela. Estraño monopolio es éste que abre las puertas del Instituto a todos los ciudadanos i les dispensa, a todos sin distincion, los tesoros de la ciencia que enseña en sus clases! 
Despojada la cuestion de todo accesorio apasionado, puede, por tanto, reducirse a estos términos sencillos: ¿Debe la Universidad abatir sus enseñanzas hasta un nivel accesible a todos o al mayor número de los alumnos que soliciten grado de Doctor, o por el contrario, cumple a los alumnos elevarse por estudios sólidos a la altura de instruccion que el Instituto considera indispensable para conceder el diploma superior de idoneidad.

No me toca en este momento inquirir si para acabar de una vez con estos constantes conflictos i dar el desarrollo conveniente a los estudios, convendria suprimir los grados académicos: lo único que me interesa hacer notar es que el grado no es un derecho que adquieren los alumnos con el hecho de inscribirse en los cursos universitarios.

Tanto los padres de familia como los educandos deberian reflexionar que los diplomas son una patente de idoneidad i un estímulo para los jóvenes, i que si los que ha espedido hasta hoi la Universidad son mas respetados i por lo mismo mas apetecidos, es porque el público no ignora que en ella es donde las pruebas son un poco mas numerosas i severas í donde se exijen mayores conocimientos para concederlos. Que se reduzcan estas garantías, i la moneda universitaria será tan despreciada en el mercado público, como la que emiten otros institutos de escasa valía.

Los superiores de la Universidad no debemos tampoco echar en olvido que los diplomas dejan de ser un estímulo desde el momento en que se conceden al charlatanismo i a la mediocridad. Los jóvenes comprenden bien sus intereses i si hoi buscan con ahínco la patente universitaria i desean alcanzarla pronto, es porque esta patente es una ejecutoria de mérito real, que el pais respeta todavía: mañana la irán a buscar a otros establecimientos de mayor nombradía o vendrán a solicitar de la Universidad que las haga ménos accesible a las nulidades, si ella cae, como empieza ya a suceder, en completo descrédito.

Si los intereses de la juventud i los de la instruccion son estrechamente solidarios, busquemos con ánimo desapasionado cuáles habrán de ser las pruebas que la universidad deberá exijir para conceder el diploma de doctor.

\section{VI}

Es bien sabido que aun en los paises donde las Universidades han funcionado sin interrupcion por un dilatado espacio de tiempo, no se han fijado todavía las ideas sobre las condiciones que deben llenar los postulantes al Doctorado. Esta incertidumbre procede de la complejidad misma de la cuestion, i ademas del carácter i de las necesidades intelectuales de cada nacion (en lo cual intervienen tambien los partidos políticos i las sectas relijiosas) i de la estension cada vez mayor que van adquiriendo las ciencias, de que resulta la necesidad de ensanchar en la misma medida los años del aprendizaje, i de subdividir las carreras profesionales.

La cuestion para nuestra Universidad es, decidir qué conocimientos deben exijirse para el grado superior en Jurisprudencia, Medicina, Injeniería o Ciencias naturales.

Es indudable que para el ejercicio mecánico de cualquiera de estas profesiones, solo se necesitan aquellas nociones que le son directa e inmediatamente aplicables. Un médico no necesita saber Gramática, ni Jeografía, ni Aritmética, ni Aljebra ni mucho ménos Cosmografía, Historia patria o Historia universal para diagnostar medianamente 
una enfermedad i para prescribir, libro en mano, la fórmula sacramental. ¿De qué le sirve a un Jurista práctico la literatura i las matemáticas, si su oficio es aplicar las leyes a los casos particulares i silo único que necesita es poseer un criterio seguro para interpretar en el sentido mas recto posible las disposiciones de los Códigos? Sinembargo, el simple sentido comun basta para discernir entre el hombre que ha recibido una cultura completa i el que ha pasado unos pocos años apénas en los bancos de los colejios.

El espíritu es como el cuerpo: necesita adquirir soltura i vigor para comprender los problemas complicados de las ciencias i para elevarse hasta aquella jeneralizacion de los fenómenos, sin la cual no hai mas que rutinas i empirismo. Así como el niño tiene que adquirir por una educacion gradual i armónica de sus miembros el temple í la flexibilidad indispensables para resistir las fatigas corporales i para llevar a cima obras duraderas i sólidas, así tambien el hombre que quiere abarcar los vastos horizontes de una ciencia ha menester de una educacion prévia i sistemática de sus facultades. ¿Qué especie de jurisconsulto seria un rústico a quien se arrancase de la escuela primaria para meterlo en el dédalo complicado de los estudios de Jurisprudencia i en la meditacion de las leyes que gobiernan el mundo moral? A tanto equivaldria esto, como obligar a un niño a ejecutar los saltos más difíciles de jimnástica o las obras mas delicadas del arte, sin haber ántes modificado la torpeza de sus músculos i la rudeza injénita de sus sentidos.

"El fin de toda enseñanza es doble, ha dicho M. Levasseur: hacer penetrar una nocion particular en la memoria, i contribuir con esta nocion a DESARROLLAR la intelijencia del niño."

En un sistema de educacion bien dirijido cada ramo dé estudio desarrolla una facultad especial de la mente: éste aviva la memoria, aquel la imajinacion i el otro despierta el espíritu de jeneralizacion o el de análisis; i todos ellos concurren a desarrollar la intelijencia i a prepararla a recibir i fecundar la semilla de las ciencias.

Es posible; diré mas, es seguro que entre los ramos que un jóven estudia durante su carrera científica o literaria, haya algunos que no tenga ocasion o necesidad de aplicar en el curso de su existencia, como es posible que no tenga necesidad de saltar un foso o de trepar por una escala de nudos, ni de levantar grandes pesos, ni de ejecutar ninguno talvez de los ejercicios que aprendió en el jimnasio del colejio; pero así como el fin que se propone la jimnástica no es el de preparar a los niños únicamente para aquellas pruebas de ajilidad, sino de dar vigor al cuerpo i ánimo al espíritu para las ulteriores luchas de la vida, así tambien el objeto de los estudios preparatorios, no es tan solo instruir a los educandos en los ramos de inmediata aplicacion a la carrera que han elejido, sino el de desarrollar su intelijencia i fortificar i dirijir aquellas facultades que habrán de aplicar más tarde al ejercicio de aquella carrera o al de cualquiera otra, ya sea manual o literaria.

"Se ha repetido mil veces, dice un ilustre institutor, que los estudios clásicos son una especie de jimnástica intelectual, que ablanda i fortifica el espíritu; que sin preparar para una carrera determinada preparan para todas; que aun cuando no deja al parecer huellas durables por el estilo de las que se comprueban con un exámen, su influencia secreta i prolongada es tal, que se distinguen fácilmente, aun en el comercio ordinario de la vida, los hombres que han hecho estudios serios i completos de los que han recibido una educacion superficial o deficiente. Hai para esto muchas razones, entre las cuales citaremos las mas jenerales: así, es fácil comprender que ocupando la juventud en las obras maestras de la literatura, en el arte de hablar i de escribir, en los grandes acontecimientos de la historia, en los grandes sucesos de la humanidad, en los grandes principios de la literatura i de la ciencia, se eleva el nivel medio de los espíritus con mas 
eficacia que por medio de estudios prácticamente mas útiles i mas directamente aplicables.

"Un comercio siquiera sea superficial, con este mundo superior e ideal, no podrá prolongarse en la edad de las impresiones vivas i fáciles sin tener influencia sobre el espíritu público i sobre los caractéres de los ciudadanos. Se comprende que cuando las clases altas de una nacion se han educado en este comercio i cuando todas las instituciones llevan la huella de las ideas adquiridas en él, las clases bajas harán esfuerzos por levantarse hasta aquel nivel; i mediante esta saludable emulacion, el fin se alcanzará tarde o temprano." (Cournot-Des institutions d'instruetion publique en France).

"La literatura i las matemáticas, dicen los señores Demogeot et Montucci en su libro sobre la educacion superior en Inglaterra, se han conservado i se conservarán por muchos años todavía, como el ámplio i sólido fundamento de la eciucacion universitaria en este pais.

"Hai en el comercio de los grandes escritores de la antigüedad, en esta razon aclamada por el sufrajio universal de los siglos, así como en el culto de la verdad absoluta incontestable e incontestada de las matemáticas, en esta "luz que ilumina a toda criatura que viene al mundo," una incomparable iniciacion para todos los trabajos del pensamiento. Oxford i Cambridge no ganarian nada i se espondrian a perder mucho si quisieran convertir a sus alumnos en especialistas, en sabios habilísimos para contar las patas de un insecto o para descubrir con el microscopio las faces de la vejetacion de un liquen, pero impotentes para conocerse a sí mismos i para conocer a sus semejantes. Tales sabios son sin duda necesarios, i sus investigaciones contribuyen a ensanchar incesantemente el patrimonio de la ciencia humana; pero ántes de entregar las jeneraciones a las carreras dejémoslas gozar un poco de la espléndida luz del dia, dejémoslas contemplar en su magnífica armonía las "bellas cosas que Dios ha hecho" o sea las leyes eternas de su divina razon, i que son el eterno substractum de todas las cosas visibles i pasajeras."

No debemos ocultar, sinembargo, que una educación clásica demasiado vasta í complicada tiene tambien graves inconvenientes que es preciso evitar entre nosotros. "El comercio con un mundo superior e ideal llena el alma de los jóvenes de pensamientos quiméricos; les prepara crueles descepciones en la transicion de la adolescencia a la virilidad, de la edad de las ilusiones jenerosas a la de las tristes realidades; despierta en muchos ambiciones a veces desordenadas, i contribuye, en los paises democráticos, a dividir la sociedad en clases. Estos peligros son en verdad ménos de temerse entre nosotros donde la educacion esclusivamente clásica ni conduce a la fortuna o a los honores, ni es del gusto de los padres de familia; i en donde no existen grandes fortunas, ni una clase bastante numerosa, capaz de comprar el primero i el mas hermoso de los lujos - una buena educacion-.

Sinembargo, entre los dos estremos, de una enseñanza clásica tan ámplia como la que se da en las universidades aristocráticas de Oxford i Cambridge, i de una yana i superficial como la que se pretende plantear, hai un medio que nosotros podemos i debemos adoptar provisoriamente.

Veamos lo que en esta materia se halla establecido en algunas de las universidades donde los estudios secundarios son un encaminamiento a los profesionales de Leyes i Medicina. 
La lei de 1858 que instituyó en Inglaterra el Consejo jeneral de educacion médica i de rejistro, establece las condiciones que ha de llenar el que aspira al título de médico competente (qualified) en el Reino Unido.

En la Universidad de Lóndres que es una de las autorizadas por aquella lei para organizar i conferir licentian at excercendum se exijen las siguientes: 1.a Que el candidato tepga por lo ménos 17 años i haya sido aprobado en el exámen de matriculacion. La Universidad exije de todos los aspirantes, sin distincion, conocimientos elementales en las ciencias i en las letras. En las ciencias deben responder a los programas respectivos, que comprenden la Aritmética i el Aljebra (comprendidas las ecuaciones de primer grado, las proporciones i las progresiones); Jeometría (comprendidos los cuatro primeros libros de Euclídes, o la materia que contienen tratada por cualquier otro autor); Mecánica (resolución de las fuerzas, máquinas sencillas); Hidrostática, Acústica i Optica; Química (comprendiendo el calor i sus efectos, i el estudio de los elementos no metálicos). En las letras, el exámen comprende el Latin, el Griego, la Historia de Inglaterra, la Jeografía moderna, la Lengua inglesa, i en fin, el Frances o el Aleman a eleecion del candidato. $2^{\bullet} a$ Pasado este exámen, que se verifica materia por materia i por un tiempo que dura a veces tres o cuatro horas para cada materia, el candidato puede presentarse al exdmen preliminar científico que abraza las materias siguientes: Mecánica, Hidráulica, Qptica, Física (calor, electricidad, magnetismo): Química, Botánica, Fisiolojía vejetal i Zoolojía, todo de acuerdo con los programas respectivos, que son, en su mayor parte, mas comprensivos que los del exámen de matriculacion.

Por no prolongar demasiado este escrito, no entro aquí en el pormenor de las pruebas ulteriores que se exijen para conferir ya sea el grado con honores o sea el doctorado, ya la simple licencia; pero esta cita basta para patentizar el hecho de que aun cuerpos puramente examinantes como la Universidad de Lóndres, que se precia de reducir sus exijencias a lo indispensable, i que se ha creado con el fin de constituir un vasto tribunal de ciencias i de literatura para toda la estension del Reino Unido, requieren para conceder el diploma de grado superior (certificate of higher proficiency) un cúmulo de materias i de garantías que parecerian en nuestro pais exhorbitantes por demas.

La creencia que existe entre nosotros de la inutilidad de tales pruebas puede provenir, o de una completa fe en la eficacia de los exámenes que desde tiempo inmemorial se estilan en nuestros Colejios i Universidades, o de que no estimamos aplicables a nuestros Institutos los reglamentos de otros paises mas avanzados, o acaso de que abrigamos la conviccion de que la vida, la fortuna i el honor de nuestros conciudadanos no merecen el interes que los institutores ingleses muestran por la vida, la fortuna i el honor de sus compatriotas.

Casi todas las Universidades de Europa, i aun las mui respetables que algunos Estados de la Union Americana subvencionan exijen iguales o mayores garantías de aptitud para conceder el diploma superior, i en todas ellas los exámenes tienen un carácter práctico i una duracion desconocidos en nuestras escuelas universitarias. Eñ éstas el exámen es casi siempre oral i no escede de 15 a 20 minutos i a las veces de 10 para cada materia, i aun para un grupo de materias; i este término queda a menudo reducido a 3 o 4 minutos, pues el resto del tiempo lo suelen emplear los examinadores en largas disertaciones i en estensas contraréplicas. La adquisicion del grado es tanto mas fácil, cuanto que cualquier individuo puede ganar todos los cursos que se requieren para obtenerlo con solo someterse a los exámenes llamados de habilitacion; i es sabido que en éstos no se obliga al candidato, como en los anuales de algunos cursos de Literatura i Filosofía i de Medicina, a escribir composiciones sobre temas dados, hacer versiones, ejecutar operaciones prácticas, en suma, a dar testimonios positivos de que posee 
realmente la materia. Los alumnos que se presentan a estos exámenes tienen, en lo jeneral, una instruccion de soplillo adquirida en pocos meses de estudio mental; raros son los que han díjerido suficientemente la materia, i los que pudieran aplicar los conocimientos, de que dan a veces muestras brillantes, a la resolucion de cualquier problema científico no incluido en el texto, pero deducible de las leyes i principios contenidos en él.

Se ve las facilidades que ofrecen a los jóvenes nuestros exámenes de habilitacion, para llegar sin mayor esfuerzo a la ansiada meta del doctorado; i no obstante esto, se habla sériamente de trabas i de monopolios i de clases privilejiadas, i se pide ahincadamente que se supriman materias i que se simplifiquen, aun mas de lo que están, los estudios, para poner el grado superior al alcance de todos o por lo ménos del mayor número de ciudadanos que lo soliciten.

\section{VII}

En mi opinion los únicos cambios que convendria hacer a los estatutos víjentes, en lo tocante al grado superior, serian la supresion de los cursos de Historia universal i de Elocucion, Retórica i Táctica de las Asambleas, en calidad de obligatorios para el grado, i su reemplazo por dos cursos elementales de ciencias naturales: Ios de Jeolojía, Química o Antropolojía, por ejemplo.

Opino que como disciplina mental i como medio de imprimir una direccion mas práctica a la enseñanza, i por consiguiente a los estudios, níngun aprendizaje es mas útil que el de las ciencias naturales. Casi no hai pais civilizado en el mundo, donde el estudio de estas ciencias no sea la base obligada de toda educacion liberal, desde la de la Escuela primaria hasta la de las grandes universidades; i nosotros mismos le hemos dado una importancia de primer órden a la enseñanza de la Zoolojía, de la Física i de la Química en las Escuelas normales i primarias de la República.

En su obra justamente célebre sobre el "Crímen i la Locura," el sabio profesor inglés Maudsley se espresa así:

"Aparte del deber positivo que todo hombre tiene de adquirir un conocimiento tan completo como le sea posible, de los objetos que le rodean con el fin de sacar partido de ellos para su desarrollo personal, el estudio i la práctica de las ciencias naturales constituyen la jimnástica mas favorable para las facultades intelectuales, porque a cada paso pone en juego la observacion, la jeneralizacion, la abstraccion í el razonamiento deductivo e inductivo. Ningún otro estudio enseña en grado igual a observar con esactitud i raciocinar con rectitud; porque en estas ciencias se busca apasionadamente la verdad por sí misma i sin mirar a lo que parece útil o inútil, o a las opiniones preconstituidas, o las pretensiones de una autoridad cualquiera.

Una conclusion no es aceptada jamas como esacta sin haber sido sometida a todos los medios posibles de verificacion...

Hai que convenir en los siguientes hechos: muchas personas son completamente incapaces de atencion sostenida, de observacion esacta i de razonamiento recto: no alcanzan a abarcar con claridad todas las faces de un problema ni a concentrar en él toda su atencion; emplean palabras sin comprender su significacion precisa; tienen creencias 
sin darse cuenta del verdadero sentido de lo que afirman; discurren sin ilacion ni coherencia sobre asuntos que se precian de conocer; creen segun sus temores, sus afectos o sus intereses, i toman por convicciones sólidamente fundadas sus preocupaciones 0 ciertos sentimientos vagos que los ajitan. Estos defectos intelectuales no son de temerse en los hombres que han adquirido algunos conocimientos en las Ciencias naturales. En este estudio hai necesidad de concentrar la atencion, de fijar con precision el sentido de las palabras, de someter, con humildad i perseverancia, el espíritu a los hechos, i de recorrer con paciencia i por grados el camino que ha conducido a otros a ciertos resultados. Aquí, el hombre que quiere saber algo tiene que constituir-se en humilde ministro e intérprete honrado de la naturaleza, o seguir las huellas de los que habiendo sido lo uno i lo otro, han formulado la ciencia; i por poco que se desvíe del verdadero método, la imperfeccion o el error serán el resultado inevitable. Siendo esto así, parece evidente que ningun estudio hai mas adecuado para fortificar i desarrollar las facultades intelectuales, puesto que no solo da el conocimiento de los hechos particulares, sino que hace contraer al espíritu el hábito precioso de observar con cuidado i de raciocinar con vigor-hábito que le servirá en sus ulteriores investigaciones. La ventaja no está, por tanto, únicamente en el poder que resulta del mayor saber, sino en un poder mas grande de adquirir el saber, pues el desarrollo intelectual marcha al compas de la evolucion de la naturaleza."

La introduccion de los estudios de Ciencias naturales entre los preparatorios para cualquier profesion liberal, fué propuesta por mí hace ya ocho años; i aunque de antigua data en el mundo, no se aceptó, seguramente porque era una idea nueva entre nosotros i no se conocia todavia su alcance. Ya ella ha hecho su camino en la educacion primaria, $i$ me parece llegado el tiempo de ponerla en práctica en la Universidad.

Se acusa a la Universidad de favorecer las tendencias especulativas de nuestra juventud i de contribuir de este modo a desviar a la sociedad de los estudios positivos i a formar una clase letrada, sin hábitos de trabajo, vanidosa i con tendencias al gamonalismo i a la empleomanía. En estas acusaciones entra por mucho el espíritu de partido, pero no por eso debemos mirarlas con absoluto menosprecio, i por el contrario cumple a la Universidad tenerlas en cuenta i esforzarse por fomentar los estudios esperimentales i por gravar profundamente en el alma de la juventud los métodos positivos de observar i de deducir.

Aceptada la reforma que indico, podría disponerse que los estudios de Historia universal i de Elocucion solo obliguen a los candidatos para el Grado superior de Jurisprudencia.

Como el comercio es la ocupacion de una escasa minoría de ciudadanos, i la Contabilidad mercantil no tiene, en realidad, aplicacion a otras industrias o profesiones, ni posee, por consiguiente, el carácter de jeneralidad o de ciencia fundamental i sujestiva, que es lo que debe buscarse en las enseñanzas preparatorias, opino que no debe incluirse entre los cursos obligatorios para el Doctorado. Acaso seria más lójico exijir el estudio de la Agricultura, que es la ocupación de la inmensa mayoría de los colombianos, o el de la Ciencia constitucional, la Lejislación i la Ciencia administrativa, que tanto ha menester una gran masa de nuestros conciudadanos (mayor sin duda que la de los comerciantes) que vive entregada al oficio de la política i deriva de él su subsistencia. 
Es lamentable sin duda que el espíritu humano no alcance su madurez completa, ni logre abarcar el conjunto de una ciencia o siquiera los principios fundamentales de ella, sino despues de largos años de incesante labor; pero a ello es preciso que cada cual se resigne de buena voluntad. La humanidad tiene que lamentar tambien la desigualdad de las fortunas i la dura necesidad impuesta a la especie de instruirse i de trabajar para llégar a la riqueza i al bienestar; pero hoi ya nadie piensa que dando un valor forzado al trabajo o a los instrumentos de cambio se pueda aumentar ni en un maravedí el capital del pobre. Aparte de la alta inmoralidad que el hecho encerraria en sí, la circunstancia de llamar la Universidad Doctor a un joven que no posee aptitudes suficientes, o la de darle el Gobierno a una moneda de plomo el valor legal de una de oro, no cambiaría en nada las condiciones del Doctor ni las de la moneda. En un país de libertad como el nuestro, cada ciudadano irá, llegado el caso, a buscar en otra parte que en la Oficina universitaria o en la Casa de moneda oficial, sus médicos, sus abogados o sus instrumentos de cambio.

El público empieza ya a comprender que la moneda universitaria tiene un feble no despreciable: si seguimos aumentándolo, nuestros Doctores no serán de recibo en ninguna parte i tendrán que ocurrir a Europa para la reacuñacion de su título.

Que la juventud acepte las fatalidades de la naturaleza, i aceptemos nosotros los encargados de guiarla, los sinsabores de nuestra posicion. La República ha encargado a la Universidad de una alta mision, i no la desempeñaria dignamente si empezara por abatir los estudios con la mira de engañar al público con diplomas falaces.

\section{IX}

El último Reglamento universitario al establecer dos especies de grados, superior e inferior, ha dado la única solucion equitativa que pudieran prometerse los jóvenes que, o por escasez de recursos o por falta de disposiciones naturales, necesitan terminar pronto una carrera i obtener una patente universitaria en que conste el grado de suficiencia que han alcanzado.

Cuando propuse esta reforma, que el doctor Santiago Pérez, Rector de la Universidad en aquella época, me hizo el honor de aceptar, tuve en cuenta las siguientes consideraciones:

1. ${ }^{a}$ La necesidad de eliminar de una vez la causa primordial de las luchas que había estado sosteniendo la Universidad, aun con los altos poderes públicos, para conservar el réjimen de sus enseñanzas i la elevacion de sus estudios. En efecto, si el anhelo de reformas se refería únicamente a las condiciones exijidas por la Universidad para conceder el diploma, parecía evidente que ofreciéndoles a los jóvenes los medios de obtener un grado ménos costoso i mas fácil, cesaría de hecho el conflicto. No cabía preveer que pudiera mas tarde solicitarse de la Universidad que rebajase los estudios hasta ese ínfimo nivel í declarase de la misma talla intelectual a los jóvenes mediocres i a los eximios.

2. ${ }^{a}$ El deber que incumbe a la Universidad de cotizar honradamente los títulos que espide. El cumplimiento de este deber era poco menos que imposible, supuesto que no existía sino un solo calificativo, i que todos los candidatos, cualquiera que fuera su mérito, se creían con derecho a obtenerlo. Grave conflicto era éste para los poderes públicos que se veían asediados constantemente por las solicitudes de influyentes padres de familia, i 
para los examinadores a quienes asaltaba el temor de cortar la carrera de muchos jóvenes.

Debía suponerse que abriéndoles una carrera a los alumnos ménos favorecidos por la naturaleza o por la fortuna, estos temores cesarían, i que las calificaciones de los exámenes representarían la verdad í nada mas que la verdad.

3. ${ }^{a}$ La necesidad de reconocer en los estatutos el hecho de la diversidad de aptitudes en los hombres. Es sabido que hai jóvenes de limitada intelijencia, que alcanzan a comprender apénas los hechos concretos de una ciencia i las leyes mas elementales de los fenómenos; pero que bien dirijidos logran poseer con suficiente perfeccion los detalles mecánicos o de inmediata aplicación a un arte o a una ciencia. Estos jóvenes llegan a ser obreros hábiles, médicos pasaderos, mecánicos meritorios o juristas entendidos, si en lugar de dedicarse a los estudios especulativos i a la comprensión de las leyes jenerales que gobiernan los hechos, se aplican a estudiar aquellos ramos de las ciencias de inmediata i directa aplicación a la profesion que han elejido. Para esta clase de educandos í para los que carecen de medios abundantes de subsistencia se ha establecido el grado de Licenciado. El grado superior se ha reservado para los jóvenes de intelijencia mas comprensiva i elevada i que poseen los medios i la capacidad suficientes para recibir una instruccion mas completa i fundamental; i a él podrán aspirar los licenciados que se sientan con fuerzas bastantes para responder al programa completo de los cursos universitarios. "El Grado superior será, segun lo espresamos en el documento aludido, el objeto de la constante ambicion de los alumnos capaces; i la noble emulacion que despertará el deseo de adquirirlo será un incentivo poderoso para inclinar a los jóvenes al estudio serio i fundamental de las ciencias."

\section{4. ${ }^{a}$ La conveniencia de multiplicar en nuestras poblaciones cierta clase}

de graduados, que por lo mismo que no han hecho grandes erogaciones para instruirse $\mathrm{i}$ obtener el título, pueden prestar sus servicios a un precio modico.

Los alumnos no han manifestado hasta hoí tendencia a aprovecharse de las ventajas que les ofrece el grado inferior; i si algunos han utilizado las disposiciones liberales relativas a la matrícula para optar a este grado, puede afirmarse que no han tenido en mira obtenerlo. El secreto de tales inscripciones es: que muchos alumnos i padres de familia abrigan la esperanza, fundada en los antecedentes del Instituto, de que en alguna de las reformas que mas tarde se harán a los Estatutos universitarios, quede establecido que los alumnos que han ganado los cursos que se exijen para el grado inferior puedan optar al superior sin necesidad de cursar otras materias. A ese término conduciría no muí tarde la simplificacion que ahora se propone.

\section{$\mathbf{X}$}

Yo suplico a los señores Catedráticos que mediten con calma en las enseñanzas de nuestra corta historia universitaria. El Instituto empezó a funcionar en 1868 bajo la inspiracion del mas puro patriotismo: entónces solo se pensaba en formar de la Universidad un centro de luces de donde salieran lejiones de jóvenes con el alma sólidamente nutrida en las enseñanzas de la ciencia i capaces de levantar al país de la postracion moral e intelectual a que lo babia conducido el prolongado abandono de las buenas enseñanzas. La preocupacion única del Instituto era estudiar los métodos mas adecuados de inculcar las ideas i de elevar los estudios al nivel de los modernos progresos: la colacion de grados se consideraba como cosa secundaria, i no se pensaba 
en cercenar los cursos, ni en reducir las enseñanzas a proporciones ridículas, ni en dar en ellas tan solo la désís que pudieran dijerir las intelijencias vulgares. Los jóvenes que en los primeros años de vida universitaria salieron de los claustros del Instituto, podian llevar con orgullo los diplomas que éste les conferia, i la reputacion de la Universidad nacional creeia en la medida en que la severidad de los exámenes i la austeridad de los pofesores se acentuaban mas i mas a los ojos del público.

El réjimen de los estudios, la dívision i el órden de los cursos, la clasificacion de las materias, el sistema de exámenes i hasta la disciplina interior del Instituto sirvieron de modelo para la organizacion de casi todas las universidades i Colejios de la República; i la influencia de la Universidad nacional se hizo sentir hasta en las Escuelas primarias.

A esa época sucedió la de un estrecho esclusivismo i de multiplicadas e injustificables reformas dictadas, no con la mira de afirmar la disciplina escolar i el réjimen de los estudios, sino con la de satisfacer exijencias indebidas o con la de contemporizar con arraigadas preocupaciones.

Todos conocemos el resultado de aquella desazon reformatoria i sabemos en qué anarquía se hallaban los estudios cuando el Congreso nacional espidió la leí que le dió ámplias facultades a la Universidad para organizarse, i a virtud de las cuales el Gran Consejo espidió el Reglamento orgánico que hoi rije al Instituto.

Penoso es confesarlo, pero esa época de relajacion i de ensayos fué tam bien de lamentable decadencia, i ninguno de nosotros podría decidir hoi silos frutos que entónces se cosecharon correspondieron a los sacrificios que la Nacion se impuso para sostener la Universidad.

No debemos olvidar quela causa del progreso se halla en el mundo entero, i en nuestro país en particular, en una situacion crítica. El oscurantismo se organiza en todas partes i funda Colejios i Cátedras i establece por donde quiera una propaganda activa contra las doctrinas consagradas por la ciencia i contra las instituciones que la dejan desarrollar en plena libertad.

La única valla contra esa propaganda es la instruccion del pueblo por medio de las Escuelas, de los Colejios i de las Universidades; pero no la mstruccion superficial, incompleta i declamatoria que tan impotente se ha mostrado en la defensa de las doctrinas i tan infecunda en la realizacion de las ideas, sino una instruccion viril i positiva que imponga respeto a las multitudes i pueda llevar la conviccion a los espíritus. Grave engaño sufren, así los que piensan que las instituciones humanas pueden sostenerse indefinidamente con espedientes o subterfujios, como los que no quieren comprender que las doctrinas que no tienen por base la conviccion están destinadas a sufrir prolongados eclipses.

La jeneracion actual, preciso es confesarlo, cuenta con muí pocos campeones capaces de sostener las buenas ideas i de imprimir una direccion saludable a los espíritus. Eduquemos sólidamente esta jeneracion si queremos que la obra del progreso, a que está consagrada la Universidad, sea durable i fecunda.

Estraña ilusion se forjan los que creen que todos los jóvenes de este tiempo solo aspiran a obtener pronto una patente de corso que les permita ejercer una industria cualquiera: no, ellos no pueden dejar de comprender que el porvenir les pertenece, í que 
la causa de la civiizacion del país está irremisiblemente condenada a zozobrar en una época no mui lejana, si no se arman con triple escudo para sostenerla dignamente.

Si por desgracia las aspiraciones de la juventud actual no se estendiesen, como algunos lo creen, mas allá de los intereses de la hora presente, a nosotros nos toca señalarle la meta e impedir que falte a su destino empequeñeciendo su espíritu i pervirtiendo su corazon. Levantémosla, que mas vale que la sociedad viva en las rejiones de lo ideal i de lo absoluto, que en el limo del individualismo estrecho i de las aspiraciones puramente materiales!

\section{$\mathbf{X I}$}

En conclusion: la Universidad necesita reformar radicalmente el réjimen de sus enseñanzas, la estructura de sus programas de estudios, el sistema de sus exámenes i la organizacion de sus Escuelas superiores. Como se ve, el señor Galan i yo hemos llegado por vías diferentes i por razones enteramente diversas, i aun opuestas, a la misma conclusíon; pero como diferimos en los medios i en los fines, propongo la siguiente modificacion a la proposicion con que termina el informe del señor Catedrático:

"El Consejo de la Escuela de Literatura i Filosofía propone a la adopción del Gran Consejo universitario la siguiente proposicion:

Nómbrese por el Gran Consejo universitario una comision plural compuesta de un Catedrático por cada escuela, que estudie el Reglamento orgánico de la Universidad, i presente al Gran Consejo, para ántes de terminar el año escolar, el plan jeneral de reformas que hubiere acordado, con un informe en que esprese las razones que motivan tales reformas. El señor Rector de la Universidad hará publicar estos documentos de manera que pueda repartirse un ejemplar impreso de ellos a cada uno de los miembros del Gran Consejo dos semanas ántes de la sesion en que deben discutírse las reformas que se propongan. En esta discusion se observará la tramitacion prescrita por el Reglamento de la Cámara de Representantes para todos los proyectos de leí."

Presentado al Consejo de Literatura i Filosofía en su sesion del día 6 de noviembre de 1878, por el infrascrito Rector de la misma.

A. VARGAS VEGA. 